\title{
Optical flare activity in the low-mass eclipsing binary GJ 3236
}

\author{
Š. Parimucha ${ }^{1}$ • P. Dubovský ${ }^{2} \cdot$ M. Vaňko ${ }^{3}$ • \\ M. Čokina ${ }^{1}$
}

Abstract We present our observations of the low-mass eclipsing binary GJ 3236. We have analyzed a phased $R_{C}$ light-curve and confirmed previously determined fundamental parameters of the components. We detected evolution of the spot(s) and found that there exists a large spot near a polar region of the primary component and another spot either on the primary or the secondary component. We also observed 7 flare events and determined a flare rate of about 0.1 flares per hour. We observed two high energy, long-term flares with a complex light curve and possibly four weak short-term flaring events. A majority of the flares was detected in the $R_{C}$ filter, which indicate their high energy.

Keywords binaries: eclipsing - binaries: low-mass stars: flares - stars: activity - stars: spots

\section{Introduction}

In what follows, the term flare will refer to violent events in stellar atmospheres, which release accumulated magnetic energy and reject hot material into space. We often observe them on magnetically active stars, and of course, on the Sun. The radiation is emitted across the whole spectrum, from $\gamma$-rays to radio

Š. Parimucha

Institute of Physics, Faculty of Science, University of P.J. Šafárik, 04401 Košice, Slovakia

P. Dubovský

Vihorlat Observatory in Humenné, 06601 Humenné, Slovakia M. Vaňko

Astronomical Institute, Slovak Academy of Sciences, 05960 Tatranská Lomnica, Slovakia

M. Čokina

Institute of Physics, Faculty of Science, University of P.J. Šafárik, 04401 Košice, Slovakia

waves. The total bolometric energy released during such a flare event can amount to $10^{24}-10^{27} \mathrm{~J}$ (Pettersen 1989).

Flares can be modeled by a beam of charged particles that are accelerated by oppositely-oriented magnetic field lines at upper levels of a stellar atmosphere. They cause acceleration of particles in the beam and propagate downward into the star's surface. During this, particles deposit energy and momentum along the way and produce electromagnetic radiation. This rapidly heats the lower stellar atmosphere, causing it to explosively expand and dramatically brighten (Allred et al. 2015). Stellar flares, however, cannot be explained by a simple scaling of solar-like flares to stars. Observations uncover problems arising from the different spectral distribution of the emitted energy, the role of the age and spectral type of the host star, the multiform magnetic field topologies on stars, the tidal forces in active binaries, etc. (Kövari et al. 2007).

Flare activity is a very common phenomenon in socalled red dwarfs - low mass, low luminous and cool stars (Davenport et al. 2012). This group of stars is the most abundant group in our galaxy. Up to date there are known only about twenty eclipsing binaries where both components are red dwarfs, because this low luminosity systems are quite difficult to uncover. Using high dispersion spectroscopy it is possible to determine basic parameters of the components with an accuracy of about $1 \%$. This is sufficient for evolutional modeling of these stars, which also play a key role in our understanding of stellar physics of main sequence stars at the bottom part of the HR diagram. Massive convection affects the significant part of the atmosphere of red dwarfs and generates strong magnetic fields, the source of high activity which manifests itself through intensive flares probably originating in the chromosphere and the corona of red dwarfs. Strong magnetic activity is also manifested by the presence of spots on the 
surface of the stars, which strongly affects the shape of light curves of eclipsing binaries containing theses stars (Irwin et al. 2009; Ribas 2003).

In this paper we present our light curve analysis and detection of optical flares in such a eclipsing system GJ 3236 (LSPM J0337+6910, 2MASS J03371407+6910498, USNO-B1.0 1591-0058025, $\alpha_{2000}$ $=03^{h} 37^{m} 14^{s} .08, \delta_{2000}=+69^{\circ} 10^{\prime} 49^{\prime \prime} .8, V=12.21 \mathrm{mag}$, $I=10.9 \mathrm{mag})$. This system was for the first time noted by Gliese and Jahreiß (1991) in the Third Catalogue of Nearby Stars. Hawley et al. (1996) included this star into a spectroscopic survey of $M$ class dwarfs in the northern hemisphere and Hünsch et al. (1999) included GJ 3236 in the catalog of nearby stars observed by the ROSAT satellite. Since then it has been listed also as an X-ray source. Eclipses on the light curve were discovered by Irwin et al. (2009), who established the ephemeris of the binary system to be

$$
H J D_{\text {min }}=2454734.99586(12)+0^{d} .7712600(23) .
$$

From radial velocity measurements of the both components the authors derived their physical properties and found that the system is binary with red dwarfs components, of the spectral type dM. The system shows signs of high activity and evidence of strong $H \alpha$ emission lines in the spectra of both components. Dittmann et al. (2014) determined trigonometric paralax for GJ 3236 to be $27.7 \pm 1.3$ marcsec, what corresponds to distance $36.1 \pm 1.7$ pc. The optical flare activity in this binary was for the first time reported by Šmelcer (2015).

\section{Observations}

Presented photometric observations were carried out at the Astronomical Observatory at the Kolonica Saddle (Kudzej et al. 2007). For all observations except the last one (March, 19. 2016) we used the Celestron C14 Edge HD telescope (aperture $356 \mathrm{~mm}$, f/11) with a Moravian Instruments G2-1600 CCD camera $(1536 \times 1024$ pixels $)$. This configuration gives us the field of view of $12^{\prime} \times 8^{\prime}$ with the resolution of 0.84 " / pixel (with $2 \times 2$ binning). The last observation was performed by the ŽIGA telescope operated by the Institute of Physics, University of P. J. Šafárik, at the same observatory. It is a Planewave CDK20 telescope (aperture $508 \mathrm{~mm} \mathrm{f/6.8)} \mathrm{with} \mathrm{a} \mathrm{Moravian} \mathrm{Instruments}$ G4-16000 CCD camera (4096×4096 pixels). This configuration gives us the field of view of $36^{\prime} \times 36^{\prime}$, with the resolution of 1.07" / pixel (with $2 \times 2$ binning).

The CCD images were reduced in the usual way (bias and dark subtraction, flat-field correction) and aperture
Table 1 The summary of our observations of the eclipsing binary GJ 3236 used in this study. The phase is given according to ephemeris (1).

\begin{tabular}{lccc}
\hline Date & Filters & Exp. time[s] & Phase \\
\hline $2015-10-30$ & $R_{C}$ & 60 & $0.701-0.336$ \\
$2015-10-31$ & $R_{C}$ & 60 & $0.987-0.607$ \\
$2015-11-01$ & $R_{C}$ & 60 & $0.297-0.579$ \\
$2015-11-03$ & $R_{C}$ & 60 & $0.872-0.254$ \\
$2015-11-05$ & $R_{C}$ & 60 & $0.699-0.112$ \\
$2015-11-24$ & $V$ & 60 & $0.128-0.451$ \\
& $I_{C}$ & 30 & $0.137-0.537$ \\
$2015-12-29$ & $V$ & 60 & $0.479-0.041$ \\
& $R_{C}$ & 30 & $0.480-0.040$ \\
$2016-02-05$ & $V$ & 60 & $0.869-0.095$ \\
& $R_{C}$ & 30 & $0.867-0.096$ \\
$2016-03-19$ & $V$ & 30 & $0.602-0.921$ \\
& $R_{C}$ & 20 & $0.603-0.920$ \\
\hline
\end{tabular}

photometry was performed using the software package C-Munipack 1 . The first-order nightly atmospheric extinction coefficients were computed from the comparison stars' measurements and the correction for the atmospheric extinction was applied to these observations.

To improve our photometric precision and eliminate possible variability of the comparison star(s), we used a multiple comparison star method (Kim, Andronov and Jeon 2004) as implemented in the package MCV (Andronov and Baklanov 2004) and computed instrumental differential magnitudes according to the main comparison star GSC 43270784, $(V=12.67, B=13.49, R=12.05, I=11.47)$ (Zacharias et al. 2015). We did not perform any correction to the international system, because a majority of our observations were carried out only in one photometric passband. But we made night-to-night systematic corrections using the TFA method (Kovacs, Bakos and Noyes 2005) implemented in the VARTOOLS Light Curve Analysis Program (Hartman et al. 2008). The average accuracy of our measurements achieved $0.018 \mathrm{mag}$ in the $V$ filter, $0.007 \mathrm{mag}$ in $R_{C}$ and $0.006 \mathrm{mag}$ in $I_{C}$ filters, respectively.

A list of our observations of the GJ 3236 system used in this study, with basic information, about each night, is presented in Table 1

\section{Light curve of GJ 3236}

The first, and up to now only one, light curve solution of GJ 3236 was given by Irwin et al. (2009), who analyzed photometric observations from the MEarth

${ }_{1}^{1}$ http://c-munipack. sourceforge.net/ 
Table 2 Photometric parameters of GJ 3236 and their statistical $1 \sigma$ uncertainties obtained from two light-curve solutions (see the text). Values denoted by the superscript ${ }^{f}$ are fixed during solutions. $T_{1,2}$ - the temperatures of the components, $\Omega_{1,2}$ - their surface potentials, $i$ - the orbital inclination, $q$ - the mass ratio, $\theta, \phi, r, k$ - the longitude, latitude, radius and temperature factor of the spots, $\sum w \chi^{2}$ - the weighted sum of squares of residuals.

\begin{tabular}{lcc}
\hline Parameter & Solution 1 & Solution 2 \\
\hline$T_{1}[\mathrm{~K}]$ & $3341 \pm 86$ & $3346 \pm 89$ \\
$T_{2}[\mathrm{~K}]$ & $3235 \pm 86$ & $3241 \pm 82$ \\
$\Omega_{1}$ & $8.68 \pm 0.12$ & $8.71 \pm 0.11$ \\
$\Omega_{2}$ & $9.73 \pm 0.11$ & $9.74 \pm 0.11$ \\
$i\left[^{\circ}\right]$ & $83.1 \pm 0.3$ & $83.1 \pm 0.3$ \\
$q$ & \multicolumn{2}{c}{$0.746^{f}$} \\
\hline Spots: & \multicolumn{2}{c}{} \\
\hline$\theta_{P, 1}\left[^{\circ}\right]$ & $107 \pm 14$ & $109 \pm 13$ \\
$\phi_{P, 1}\left[^{\circ}\right]$ & $14 \pm 7$ & $15 \pm 8$ \\
$r_{P, 1}\left[^{\circ}\right]$ & $62 \pm 19$ & $66 \pm 20$ \\
$k_{P, 1}$ & $0.9^{f}$ & $0.9^{f}$ \\
$\theta_{P, 2}\left[^{\circ}\right]$ & - & $11 \pm 11$ \\
$\phi_{P, 2}\left[^{\circ}\right]$ & - & $54 \pm 9$ \\
$r_{P, 2}\left[^{\circ}\right]$ & - & $27 \pm 16$ \\
$k_{P, 2}$ & - & $0.9^{f}$ \\
$\theta_{S, 1}\left[^{\circ}\right]$ & $184 \pm 15$ & - \\
$\phi_{S, 1}\left[^{\circ}\right]$ & $92 \pm 7$ & - \\
$r_{S, 1}\left[^{\circ}\right]$ & $44 \pm 21$ & - \\
$k_{S, 1}$ & $0.9^{f}$ & - \\
\hline$\sum w \chi^{2}$ & 0.1538 & 0.1502 \\
\hline
\end{tabular}

project (Nutzman and Charbonneau 2008), together with their own radial velocity measurements of the both components (see Figures 2 and 3 in their paper). They found that GJ 3236 is a low-mass binary with the component masses of $0.38 M_{\odot}$ and $0.28 M_{\odot}$, and with the stellar radii of $0.38 R_{\odot}$ and $0.30 R_{\odot}$, which are about $10 \%$ larger than the theoretical predictions. This is in agreement with the results obtained for known low-mass binaries and is mostly explained by large areas of dark spots on the surfaces of both components, which decrease the stars' radiating surfaces and also yield a smaller effective temperature and a larger radius (Chabrier. Gallardo and Baraffe 2007). Irwin et al. (2009) modeled their light curve by cool, close to polar spots on the surface of each component.

Our $R_{C}$ light curve (see Fig 1.) is markedly different from the MEarth ones and cannot be fitted by the model from Irwin et al. (2009). A detrended light curve is quite stable during the interval of our observations up to changes (about $0.03 \mathrm{mag}$ ) in the phase interval 0.5 - 1.0. In this interval two large flares occurred (see Section 3), so we concluded that these night-to-night variations are connected with flare activity.
We removed flare events from the phased light curve (see Section (4) and analyzed it with the PHOEBE package (Prša and Zwitter 2005). As initial parameters for the components temperatures, surface potentials and orbital inclination we adopted the values from Irwin et al. (2009). The mass ratio $q$ was fixed to be 0.746 as a result of radial velocity solution.

The observed variations of the light curve can be explained by the presence of the spots. We have tested several different configurations of spots' locations, dimensions and temperatures. The light curve shape in the phase interval of $0.0-0.5$ at the first sight suggests presence of a hot spot on the surface of one of the components. But the solutions with hot spot(s) on the primary and/or secondary component in combination with one or more cool spots were not sufficient, mainly because of the inadequate depth of the primary minimum, although the secondary minimum and out of minima part were fitted quite well. So we reject this solution as not being a proper one.

To explain the depth of the primary minimum, as well as variations on the light curve, we have two possible solutions. If we assume that the primary minimum is caused by the passage of secondary (smaller and less massive) star in front of the primary (large, more massive) star, we need to reduce the light from system during this passage. This could be done by:

1. a cool spot placed on the secondary component on the hemisphere visible during the minimum and another spot close to the polar region of the primary component visible mainly in the phase interval of $0.5-1.0$.

2. two cool spots on the primary component, one close to the polar region as in the previous case, and the second one visible during the primary minimum.

During solution of the light curve, we fixed the temperature factor of the spots and varied their positions (longitude and latitude) and radii. We also varied components' temperatures, surface potentials and orbital inclination. These values did not change significantly from the solution of Irwin et al. (2009) and did not have strong influence on the model light curve.

The PHOEBE package gives formal statistical errors of the fitted parameters determined from the covariance matrix. These values are often underestimated. To obtain more realistic robust statistical uncertainties of the fitted parameters we used the Monte Carlo simulation method (Press et al. 2002). We assumed that the probability distribution of our measurements' errors is normal (Gaussian). We produced 10000 synthetic LCs around the best solution and fitted them using capabilities of the PHOEBE-Scripter to obtained set of parameters and corresponding $\chi^{2}$. Statistical errors of 


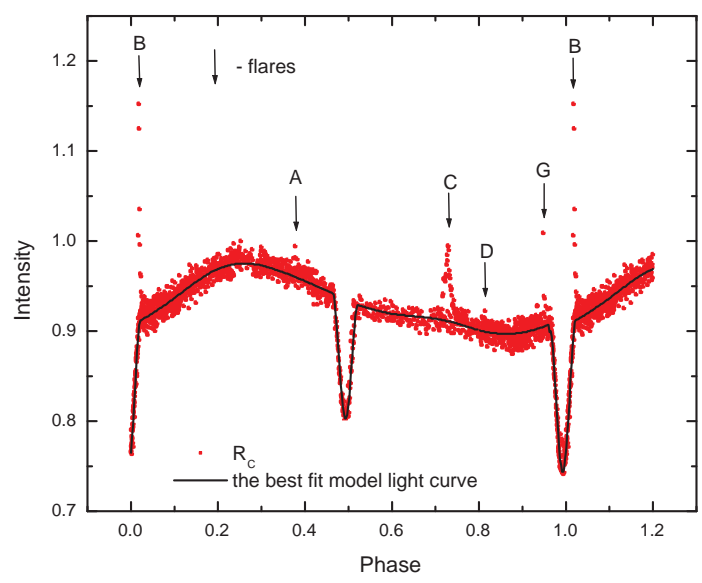

Fig. 1 The phase light curve of GJ 3236 obtained from our $R_{C}$ observations according to ephemeris (1). We also denote detected flares in the $R_{C}$ passband (see Table 3) and give the best-fit-model light curve for Solution 2 (see the text).

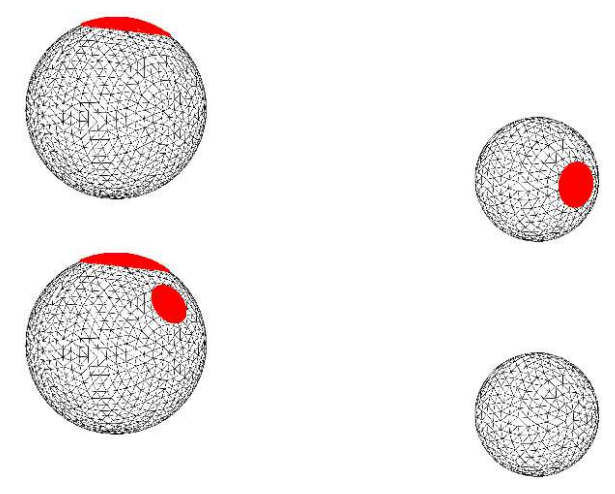

Fig. 2 A model of components in GJ 3236 with spots' locations (see the text) for Solution 1 (top) and Solution 2 (bottom).

individual parameters were established as a $1 \sigma$ confidence interval of the projection of an 11-dimensional parameters space.

In Table 2 we present results for both spotted solutions. Our results show that Solution 2 has a smaller weighted sum of squares of residuals, but without other type of observations (e.g. high resolution spectroscopic Doppler imaging) we cannot conclude which model is correct. Our photometric solution did not markedly change determination of absolute parameters of the components from Irwin et al. (2009) and all values can be considered equal within the errors.

The phase light curve of GJ 3263, together with the best fit model light curve for Solution 2, is depicted in Fig 1. Fig 2 shows the configuration of the components with spots locations listed in Table 1.

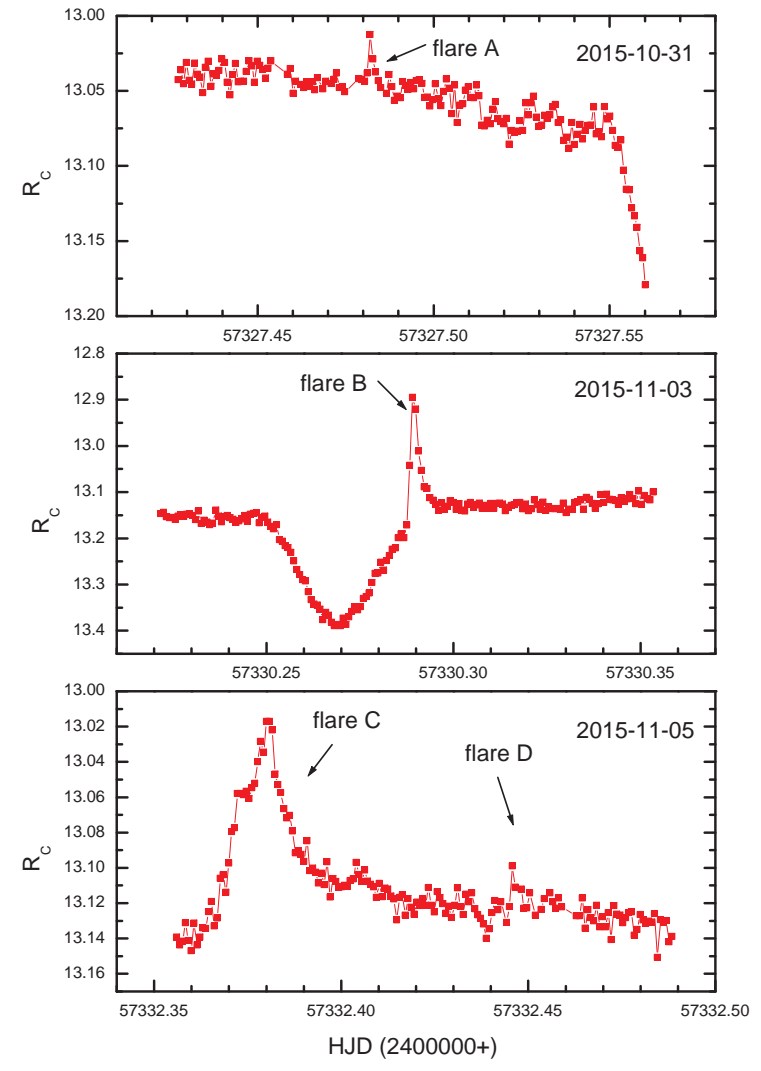

Fig. 3 Flares detected in the $R_{C}$ passband at different nights. For the parameters of the flares, see Table 3

\section{Flare activity}

GJ 3236 was selected as one of the targets of The Dwarf Project (Pribulla et al. 2012), which is focused at detection of sub-stellar companions to low-mass (composed of late-type, sD or/and WD components) detached eclipsing binaries using minima timing. Our observations were from the beginning focused mostly on determination of primary minima times and we did not gather regularly all light curves. This considerably restricted our chance to detect any flares. So we do not list these observations in Table 1

We used two criteria for flare detection: (1) a flare should last for several minutes - at least 3 times longer than the used exposure time for a CCD image, (2) at least two successive data points on the light curve are brighter (within their errors) than the quiescent surrounding of the fitted light curve plus $3 \sigma$ level. The light curve around potential flare was fitted by the $3^{r d}$ order weighted polynomial function. Weights were selected as $1 / e_{i}^{2}$, where $e_{i}$ are magnitude errors of individual points determined from photometry. The start and 
Table 3 Flares detected in GJ 3236 and their parameters: Label - designation of the flare, Date - the date of observation, Start, End - estimates when the flare started or finished, Maximum - the time of maximum brightness, Maximum phase the phase according to ephemeris (1) of maximum brightness, $\Delta \mathrm{m}$ - the brightening amplitude, Duration - the duration of the flare, Energy - estimated energy with the error in parenthesis (for discussion about energy see Section 5).

\begin{tabular}{ccccccccc}
\hline Label & Date & Start & $\begin{array}{c}\text { Maximum } \\
\text { HJD }\end{array}$ & End & $\begin{array}{c}\text { Maximum } \\
\text { phase }\end{array}$ & $\Delta \mathrm{m}$ & $\begin{array}{c}\text { Duration } \\
{[\mathrm{min}]}\end{array}$ & $\begin{array}{c}\text { Energy } \\
{\left[10^{25} \mathrm{~J}\right]}\end{array}$ \\
\hline A & $2015-10-31$ & 57327.4799 & 57327.4819 & 57327.4841 & 0.378 & $0.04\left(R_{C}\right)$ & 6.1 & $1.1(7)$ \\
B & $2015-11-03$ & - & 57330.2891 & 57330.2972 & 0.023 & $0.24\left(R_{C}\right)$ & $>10$ & $>4.8(5)$ \\
C & $2015-11-05$ & 57332.3663 & 57332.3803 & 57330.3987 & 0.729 & $0.10\left(R_{C}\right)$ & 46.6 & $5.7(5)$ \\
D & & 57332.4447 & 57332.4457 & 57330.4494 & 0.814 & $0.03\left(R_{C}\right)$ & 6.7 & $0.8(7)$ \\
E & $2015-11-24$ & 57351.4182 & 57351.4232 & - & 0.419 & $1.39(V)$ & $>35$ & $>17.4(9)$ \\
E & & 57351.4176 & 57351.4238 & - & 0.420 & $0.19\left(I_{C}\right)$ & $>35$ & $>10.1(9)$ \\
F & $2016-02-05$ & 57424.3038 & 57424.3048 & 57424.3093 & 0.917 & $0.10(V)$ & 7.9 & $1.2(3)$ \\
G & & 57424.3270 & 57424.3283 & 57424.3336 & 0.947 & $0.44(V)$ & 9.5 & $5.3(7)$ \\
G & & 57424.3278 & 57424.3289 & 57424.3327 & 0.948 & $0.12\left(R_{C}\right)$ & 7.1 & $1.6(6)$ \\
\hline
\end{tabular}

the end of the flare was determined visually according to the fitted light curve, brightnennig $\Delta \mathrm{m}$ was calculated as the difference between the maximum brightness and the fitted light curve.

The first flare event (labeled A) was observed on October 31, 2015, with the maximum at the phase of $\sim 0.38$ with the amplitude of about $0.04 \mathrm{mag}$ in the $R_{C}$-filter and the duration of about 6 minutes. It is a disputable flare, because it was on the edge of our detection criteria, which is caused by quite poor night quality, but brightening is clearly visible on the light curve (see Fig 3, top) The next night no flares were detected within the observational errors and detection criteria.

The evident flare (B) was detected just after the primary minimum on November 3, 2015, (see Fig 3, middle) at the phase of 0.023 . We cannot exactly determine when this flare started, although we can see no distortion of the minimum light curve. It means that the flare started probably shortly at the end of the minimum or just after it. The amplitude reached $0.24 \mathrm{mag}$ in the $R_{C}$-filter and the flare lasted more than 10 minutes.

Another two flares (C and D) occurred on November 5, 2015, (see Fig 3, bottom) at the phase of 0.734 resp. 0.814. Duration of the first one was more than 46 minutes with the amplitude of $0.10 \mathrm{mag}$ in the $R_{C^{-}}$ filter. The second one was a short-term (6.7 minutes) and low-amplitude (0.03 mag in the $R_{C}$-filter) flare.

On November 24, 2015, a strong flare (E) was detected simultaneously in $V$ and $I_{C}$ passbands (see Fig 4 , top). The flare lasted more than 35 minutes at the phase of 0.421 and its amplitude eas $1.39 \mathrm{mag}$, resp. $0.19 \mathrm{mag}$ in $V$, resp. $I_{C}$-filters.

Another two flares ( $F$ and $G$ ) were observed on February 5, 2016. The first one was detected only in the $V$-filter at the phase of 0.917 , lasted $\sim 8$ minutes with the amplitude of 0.1 mag. The second flare was detected in the $V$ and $R_{C}$-filters at the phase of 0.947 .
The flare in $V$ lasted more than 9 minutes and the amplitude was 0.44 mag., while the $R_{C}$ flare lasted 7 minutes with the amplitude of $0.12 \mathrm{mag}$.

No flares were detected during our last observation on March 19, 2016.

We tried to roughly determine the energy emitted by the flares. We assumed bolometric quiescent luminosities of the components determined by Irwin et al. (2009) $\left(L_{1}^{b o l}=0.016 L_{\odot}, L_{2}^{b o l}=0.009 L_{\odot}\right)$ and the Planck law of the energy distribution of the flare emission. Quiescent luminosity of a star with the radius $R$ in the specific filter $\mathfrak{F}$ can be estimated as:

$L(\mathfrak{F})=\int_{0}^{\infty} 4 \pi R^{2} S_{\mathfrak{F}}(\lambda) F(\lambda) d \lambda$,

where $S_{\mathfrak{F}}(\lambda)$ is the transmission curve of the filter and $F(\lambda)$ is the out-of-flare flux of the star at the wavelength $\lambda$. The total emitted energy by the flare in the filter is calculated by

$E(\mathfrak{F})=L_{1+2}(\mathfrak{F}) \int_{t_{B}}^{t_{E}}\left(10^{-0.4 \Delta m(t)}-1\right) d t$,

where we integrated from the beginning $t_{B}$ to the end $t_{E}$ of the flare, $L_{1+2}(\mathfrak{F})$ denotes the total luminosity of the binary and $\Delta m(t)$ is the magnitude evolution of the flare with time. Properties of all detected flares are summarized in Table 3 .

\section{Discussion and conclusion}

Our observations of GJ 3236 showed that this low-mass system is a very active binary. The solution of our $R_{C}$-light-curve confirmed previously determined values for the components' radii and masses by Irwin et al. (2009). But the light curve has significantly changed since Irwin's observations. This can be explained by the 
6

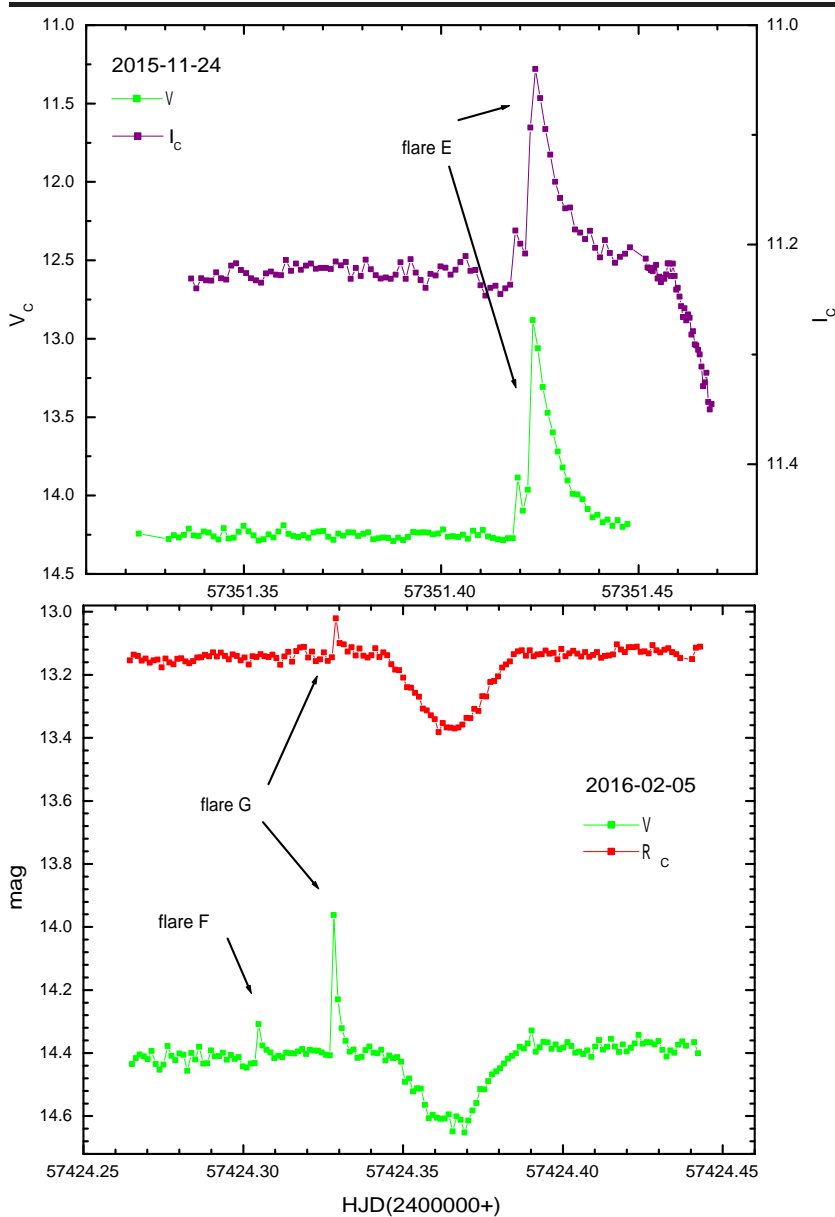

Fig. 4 Flares detected in $V, I_{C}$ (top) and $V, R_{C}$ (bottom) at 2 different nights. For the parameters of the flares see, Table 3 .

evolution of the spot(s) on the components. We found that there probably exists one large spot or a large area of many smaller spots close to the pole of the primary component. The radius of this spot or area is about 1.5 times larger than in 2008 (the year of MEarth observations). Another spot should exist on the primary, or on the secondary component. Unfortunately, we cannot decide without additional, mainly spectroscopic observations, which spot's solution is correct.

Such big spots suggest enormous magnetic activity of the system and one can expect flare events. We have photometrically monitored GJ 3236 for more than 65.7 hours and detected 7 flare events, which revealed a flare rate of about 0.1 flares per hour assuming an even time distribution of the flares. This is similar to flare rate of DV Psc, 0.082 flares per hour (Pi et al. 2014), and about two times more than in other similar systems CU Cnc, 0.05 flares per hour (Qian et al. 2012) and CM Dra, 0.057 flares per hour (Nelson and Caton 2007).

The flares are detected mainly in the short wavelength passbands $(U$ and $B)$. The fact that we observe flares in $R_{C}$ and even $I_{C}$-filters suggests their high energy. The energy determined in the specific passbands as given in Table 3 should be considered as the lower limit, mainly because of the uncertain time interval of the flare, its amplitude and out of flare level of the light curve. We also do not take into account spots for luminosity determination of the components. Moreover detection of a flare depends not only on its energy, but also on its position with respect to the observer. No information about positions of flares can be obtained from the photometry. Positions of all detected flares are uncertain, except for the most energetic flare E, which probably occurred on the primary component, given the phase when it was observed and the system geometry. We should conclude that more realistic values of the emitted energy are more than one order higher than the values given in Table 3 According to the review of Pettersen (1989) we can estimate bolometric flare energy to be $\sim 10^{27} \mathrm{~J}$.

Photometric precision and the time resolution of our observations do not allow us to observe weak flaring events. Only two such low energy flares in $R_{C}$ (flares $\mathrm{A}$ and $\mathrm{D}$ ) and one in $V$ (flare F) passbands were detected using our detection criteria. Flare $\mathrm{G}$ can be also regarded as a low energy short-term flare. These types of flares last not more than a few, up to 10 minutes and no signs of any structure on the LCs are visible. This is only a few points over detection limit.

More energetic flares $\mathrm{C}$ and $\mathrm{E}$ lasted several tens of minutes and the shapes of their LCs are more complicated. On the rising part of the LCs we can detect small brightness decrease (flare E) or a short plateau (flare C) followed by steep brightening. The decreasing part of the LC could be approximated by an exponential decay. Davenport et al. (2014) showed, from analysis of Kepler observations of GJ 1243, that such a complex behavior of flares is very common for long lasting flares with duration more than 50 minutes. That is in agreement with our observations, although our determination of flares duration is uncertain. This complex behavior of flares could be connected with physical processes linked with active regions and magnetic energy dissipation or it can be explained by a random superposition of different flares from separate regions. To reveal the rate, properties and nature of the complex, as well as lowenergetic flares, one need to observe them in at least two passbands with high time resolution.

Acknowledgements This work has been supported by the project APVV-15-0458 of the Slovak Research and Development Agency. M.V. would like to thank the project VEGA 2/0143/14. 


\section{References}

Allred, M. C., Kowalski, A.F., Carlsson, M.: Astrophys. J., 809, 104 (2015)

Andronov, I. L., \& Baklanov, A. V.: Astron. School Rep., 5, $264(2004)$

Chabrier, G., Gallardo, J., Baraffe, I.: Astron. Astrophys. 472, 17 (2007)

Davenport, J.R.A.,et al.: Astrophys. J. 748, 58 (2012)

Davenport, J.R.A., et al.: Astrophys. J. 797, 122 (2014)

Dittmann J.A., et al.: Astrophys. J. 784, 156 (2014)

Gliese, W., Jahreiß, H.: Preliminary Version of the Third Catalogue of Nearby Stars (1991)

Hartman, J.M., et al.: Astrophys. J. 675, 1254 (2008)

Hawley, S.L., Gizis, J.E., Reid, I.N.: Astron. J. 112, 2799 (1996)

Hünsch, M., et al.: Astron. Astrophys. Suppl. Ser. 135, 319 (1999)

Irwin, J., et al.: Astrophys. J. 701, 1436 (2009)

Kim, Y.,Andronov, I.L., Jeon, Y.B.: Journal of Astronomy and Space Sciences 21, 191 (2004)

Kovacs, G., Bakos, G., Noyes, R.W.:Mon. Not. R. Astron. Soc.356, 557 (2005)

Kudzej, I., et al.: Odessa Astronomical Publications 20, 100 (2007)

Kövari, Z., et al.: Astron. Nachr. 328, 904 (2007)

Nelson, T.E., Caton, D.B.: Inf. Bull. Var. Stars 5789 (2007)

Nutzman, P., Charbonneau, D.: Publ. Astron. Soc. Pac. 120, 317 (2008)

Pettersen, B. R.: Solar Physics 121, 299 (1989)

Pi, Q., Zhang, L.Y., Zhong-mu, L., Zhang, X.: Astron. J. 147, 50 (2014)

Press W. H., Teukolsky S. A., Vetterling W. T., Flannery B. P.: Numerical recipes in C, Cambridge University Press, London (2002)

Pribulla, T., et al.: Astron. Nachr. 333, 754 (2012)

Prša, A., Zwitter, T.: Astrophys. J. 628, 426 (2005)

Ribas, I. 2003, Astron. Astrophys. 398, 239 (2003)

Qian, S.B., Zhang, J., Zhu, L.Y., at al.: Mon. Not. R. Astron. Soc. 423, 3646 (2012)

Šmelcer, L.: Open Europen Journal on Variable Stars 169, $14(2015)$

Zacharias, N., Finch, C., Subasavage J., et al.: Astron. J. 150, 101 (2015) 